\title{
A human class II MHC-derived peptide antagonizes phosphatidylinositol 3-kinase to block IL-2 signaling
}

\author{
Michelle L. Boytim, ${ }^{1}$ Pamela Lilly, ${ }^{2}$ Katerina Drouvalakis, ${ }^{2}$ Shu-Chen Lyu, ${ }^{3}$ \\ Ron Jung, ${ }^{3}$ Alan M. Krensky, ${ }^{1,2}$ and Carol Clayberger ${ }^{1,2,3}$ \\ ${ }^{1}$ Program in Immunology, \\ ${ }^{2}$ Division of Immunology and Transplantation Biology, Department of Pediatrics, and \\ ${ }^{3}$ Department of Cardiothoracic Surgery, Stanford University, Stanford, California, USA \\ Address correspondence to: Carol Clayberger, Department of Cardiothoracic Surgery, Stanford University, Stanford, \\ California 94305-5407, USA. Phone: (650) 725-3279; Fax: (650) 725-3846; E-mail: cclay@leland.stanford.edu.
}

Received for publication August 12, 1999, and accepted in revised form March 28, 2000.

\begin{abstract}
MHC molecules bind antigenic peptides and present them to $\mathrm{T}$ cells. There is a growing body of evidence that MHC molecules also serve other functions. We and others have described synthetic peptides derived from regions of MHC molecules that inhibit T-cell proliferation or cytotoxicity in an allele-nonspecific manner that is independent of interaction with the T-cell receptor. In this report, we describe the mechanism of action of a synthetic MHC class II-derived peptide that blocks T-cell activation induced by IL-2. Both this peptide, corresponding to residues 65-79 of $\mathrm{DQA}^{*} 03011$ (DQ 65-79), and rapamycin inhibit p70 S6 kinase activity, but only DQ 65-79 blocks Akt kinase activity, placing the effects of DQ 65-79 upstream of mTOR, a PI kinase family member. DQ 65-79, but not rapamycin, inhibits phosphatidylinositol 3-kinase (PI 3-kinase) activity in vitro. The peptide is taken up by cells, as demonstrated by confocal microscopy. These findings indicate that DQ 65-79 acts as an antagonist with PI 3-kinase, repressing downstream signaling events and inhibiting proliferation. Understanding the mechanism of action of immunomodulatory peptides may provide new insights into T-cell activation and allow the development of novel immunosuppressive agents.
\end{abstract}

J. Clin. Invest. 105:1447-1453 (2000).

\section{Introduction}

Although a primary function of MHC molecules is to present antigenic peptides to T cells (1), it is now apparent that MHC molecules also function as immunoregulatory agents. Killer inhibitory receptors expressed on the surface of $\mathrm{T}$ cells and natural killer cells interact with conserved regions of MHC class I molecules on target cells to inhibit lysis $(2,3)$. MHC molecules have been implicated in prevention of graft rejection because administration of purified MHC molecules $(4,5)$ or synthetic peptides corresponding to regions of class I (6-9) or class II (6-12) MHC molecules induce graft tolerance in animal models. However, the mechanisms by which MHC molecules or peptides derived from them inhibit immune responses have not been clearly defined.

Drugs such as cyclosporin A, FK-506, and rapamycin not only prevent graft rejection but have also provided insight into the molecular mechanisms of rejection and lymphocyte activation. In particular, these drugs have been used to delineate signaling pathways in lymphocytes. Cyclosporin A was central to discovering that the phosphatase calcineurin activates the transcription factor NF-AT, which in turn is crucial for the transcription of IL-2 (13). In contrast, rapamycin affects later stages of T-cell activation and has been critical to understanding the IL-2 receptor (IL2-R) signal transduction pathway (14). We previously described a peptide correspon- ding to amino acids $65-79$ of the $D Q A * 03011$ allele (DQ 65-79) that has antiproliferative effects on peripheral blood lymphocytes, particularly T cells. A second peptide, designated DQ 65-79(72D), is substituted at position 72 with an aspartic acid residue for asparagine. This peptide does not exhibit the inhibitory effects of DQ 65-79 (15). Both DQ 65-79 and rapamycin block IL-2-mediated cell-cycle progression through inhibition of CDK2 kinase activity and prolonging the expression of the cell-cycle inhibitor protein $(15,16)$.

To gain a better understanding of the mechanism of action of DQ 65-79, its effects on the IL-2R signaling cascade were examined. IL-2 activates multiple pathways involving numerous molecules. Based on our previous work, we focused on the portion of the pathway inhibited by both rapamycin and the peptide (17) (Figure 1). Rapamycin, when bound to FK-506 binding protein (FKBP), blocks this pathway by inhibiting the activity of mTOR (14). In this report we define the intervention point of DQ 65-79 in the IL-2R signaling cascade, and differentiate the effects of DQ 65-79 from those of rapamycin.

\section{Methods}

Peptides, antibodies, and reagents. Peptides were prepared as described (15). Rapamycin was a gift from Wyeth-Ayerst Laboratories (Philadelphia, Pennsylvania, USA). Wort- 
mannin was purchased from Calbiochem-Novabiochem Corp. (San Diego, California, USA). Ab's specific to p70 S6 kinase, Fyn, and Lck were purchased from Santa Cruz Biotechnology Inc. (Santa Cruz, California, USA). Ab's to phosphotyrosine (4G10), Jak1, Jak3, Fyn, Lck, Akt, $\mathrm{p} 85$, and $\mathrm{p} 110-\beta$ were purchased from Upstate Biotechnology Inc. (Lake Placid, New York, USA). Ab's specific to IL-2R $\alpha$, IL-2R $\beta$, and IL-2R $\gamma$ were purchased from PharMingen (San Diego, California, USA). Unless specified, all other reagents were purchased from Sigma Chemical Co. (St. Louis, Missouri, USA).

Peripheral blood lenkocyte and T-cell isolation and cell culture. Peripheral blood leukocyte isolation and T-cell purification were done as described (18). Cells were cultured in RPMI 1640 supplemented with 10\% FCS, $100 \mathrm{U} / \mathrm{mL}$ penicillin/streptomycin, $2 \mathrm{mM}$ L-glutamine, and $10 \mathrm{mM}$ HEPES. IL-2-dependent $T$ cells were prepared by stimulating T cells with phytohemagglutinin $\mathrm{P}(5 \mu \mathrm{g} / \mathrm{mL})$ for 72 hours. The cells were then cultured with $100 \mathrm{U} / \mathrm{mL}$ IL-2. Once a week, the cells were restimulated with phytohemagglutinin $\mathrm{P}(0.8 \mu \mathrm{g} / \mathrm{mL})$. In experiments using wortmannin, cells were cultured in AIM-V medium (Life Technologies Inc., Gaithersburg, Maryland, USA).

Immunoprecipitation and Western blot. IL-2-dependent T cells $\left(1 \times 10^{7}\right.$ to $\left.2 \times 10^{7}\right)$ were washed twice with PBS (pH 6.5) and incubated for 3 hours in RPMI 1640 containing $0.5 \%$ human $\mathrm{AB}$ serum. This was followed by incubation with peptide $(40 \mu \mathrm{M})$, rapamycin $(100 \mathrm{nM})$, or wortmannin $(100 \mathrm{nM})$ for 30 minutes before stimulation with $1,000 \mathrm{U} / \mathrm{mL} \mathrm{IL-2}$ for 15 minutes. After treatment, cells were recounted, lysed, and subjected to immunoprecipitation and blotting as described (15).

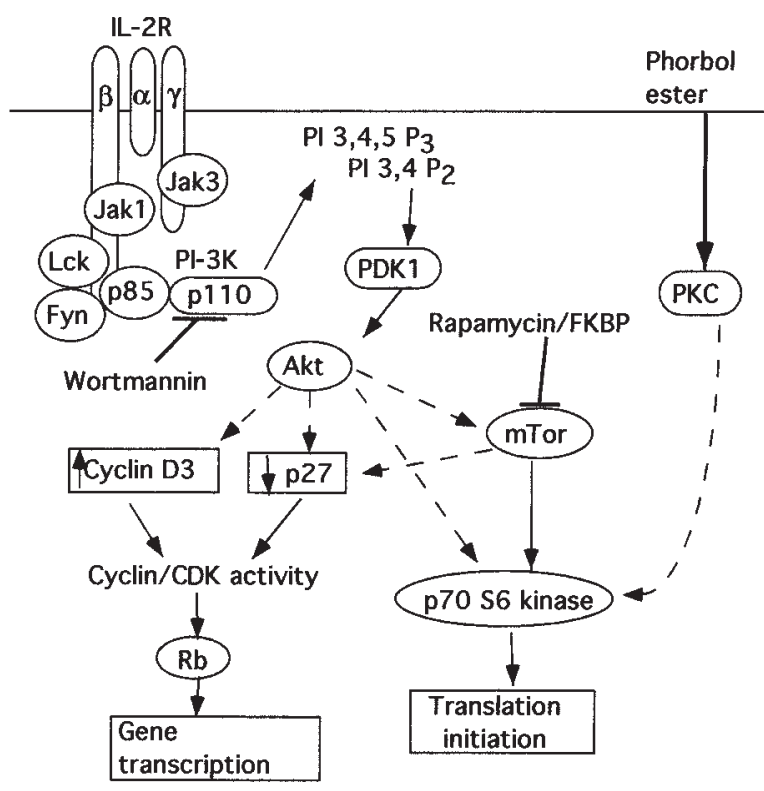

Figure 1

A simplified model depicting the pathways leading from the IL-2R to cellcycle progression (adapted from ref. 17). Pathways that may be indirect are shown with dashed arrows. PI-3K, PI 3-kinase; Rb, retinoblastoma protein; PKC, protein kinase C; FKBP, FK-506 binding protein.
Fyn kinase and Lck kinase assays. IL-2-dependent T cells $\left(5 \times 10^{6}\right.$ per condition) were activated with IL-2 as described above. Cells were lysed and kinase assays were performed essentially as described (19).

Assay for p70 S6 kinase. IL-2-dependent T cells $\left(10^{7}\right.$ per condition) were activated with IL-2 in the presence of various agents as described above for immunoprecipitation. The samples were divided into two equal aliquots, lysed in buffer B (0.1\% NP-40, 2 $\mathrm{mM}$ DTT, $50 \mathrm{mM} \beta$-glycerophosphate, $10 \mathrm{mM}$ $\mathrm{KH}_{2} \mathrm{PO}_{4}, 1 \mathrm{mM}$ EDTA, $5 \mathrm{mM}$ EGTA, $10 \mathrm{mM} \mathrm{MgCl}$, $40 \mu \mathrm{g} / \mathrm{mL}$ PMSF, and $1 \mathrm{mM} \mathrm{Na}_{3} \mathrm{VO}_{4}$ ), and immunoprecipitated with anti-p70 S6 kinase. One aliquot was subject to SDS-PAGE and Western blotting as described above. The other aliquot was processed for kinase activity. The immunoprecipitated kinase was washed twice in buffer $B$ and then washed twice in 10 $\mathrm{mM} \beta$-glycerophosphate, $10 \mathrm{mM} \mathrm{MgCl} 2,10 \mu \mathrm{g} / \mathrm{mL}$ leupeptin, $0.4 \mathrm{mM}$ DTT, and $20 \mathrm{mM}$ Tris ( $\mathrm{pH} 7.5$ ). The kinase buffer $(40 \mu \mathrm{L})$ contained $100 \mu \mathrm{M}$ ATP, 200 $\mu \mathrm{Ci} / \mathrm{mL}\left[\gamma^{32} \mathrm{P}\right] \mathrm{ATP}$ (Amersham Life Sciences Inc., Arlington Heights, Illinois, USA) and $125 \mu \mathrm{M}$ S6 peptide (Santa Cruz Biotechnology Inc.). The reaction mixture was incubated for 15 minutes at $30^{\circ} \mathrm{C}$, and then the reaction was terminated by adding $40 \mu \mathrm{L}$ of ice-cold $20 \mathrm{mM}$ EDTA ( $\mathrm{pH}$ 8.0). Triplicate aliquots $(10 \mu \mathrm{L})$ were spotted onto phosphocellulose membranes (Life Technologies Inc.). Membranes were immersed briefly in a $1 \% \mathrm{H}_{3} \mathrm{PO}_{4}$ solution containing $10 \mathrm{mM} \mathrm{Na}_{4} \mathrm{P}_{2} \mathrm{O}_{7}$, washed in $1 \% \mathrm{H}_{3} \mathrm{PO}_{4}$, and quantitated by liquid scintillation counting.

Akt kinase assay. IL-2-dependent $\mathrm{T}$ cells $\left(10^{7}\right.$ per condition) were activated with IL-2 as described above. Akt kinase assay was performed using a kit from Upstate Biotechnology Inc.

Phosphatidylinositol 3-kinase assay. IL-2-dependent T cells $\left(5 \times 10^{6}\right.$ per condition) were activated with IL-2 as described above. Cells were lysed, and the phosphatidylinositol 3-kinase (PI 3-kinase) assay was performed as described (20). For experiments in which the peptide was added directly to the kinase assay, cells were prepared without peptide treatment before immunoprecipitation. Either the peptide $(40 \mu \mathrm{M})$, rapamycin $(100 \mathrm{nM})$, or wortmannin $(100 \mathrm{nM})$ was added to the kinase reaction at the same time as the lipid substrate.

Cell staining and confocal microscopy. A CD8 ${ }^{+}$long-term cytotoxic T-lymphocyte line $\left(10^{6}\right.$ cells $\left./ \mathrm{mL}\right)$ was incubated in RPMI 1640 supplemented with DMSO or DQ 65-79-IRS (NIAVLKHNLNIVIKRRYIRS) $(40 \mu \mathrm{M})$ for 1 hour at $37^{\circ} \mathrm{C}$. After being washed, $20 \mu \mathrm{L}$ of cell culture $\left(10^{6}\right.$ cells $\left./ \mathrm{mL}\right)$ was placed into wells of glass slides precoated with poly-L-lysine and incubated for $15 \mathrm{~min}$ utes. Cells were then washed, and fixed with $4 \%$ paraformaldehyde in PBS ( $\mathrm{pH}$ 7.4). Cells were permeabilized and blocked with $20 \mu \mathrm{L}$ of $5 \%$ human $\mathrm{AB}$ serum, $5 \%$ goat serum, $0.1 \%$ NP-40, $0.01 \%$ saponin (Calbiochem-Novabiochem Corp.), and 1\% milk in PBS. They were then washed, and incubated with antiIRS $\mathrm{mAb}\left(\mathrm{IgG}_{1}\right.$; Covance Research Products Inc., Rich- 
mond, California, USA) and anti-HLA class I mAb $\left(\mathrm{W} 6 / 32, \mathrm{IgG}_{2 \mathrm{a}}\right.$ ) for 1 hour at room temperature. After cells were washed, $20 \mu \mathrm{L}$ of secondary antibodies (biotin-conjugated anti-mouse $\operatorname{IgG}_{1}$ and FITC-conjugated anti-mouse $\mathrm{IgG}_{2 \mathrm{a}}$ from PharMingen) were added, followed by streptavidin Alexa Fluor 594 conjugate and anti-fluorescein Alexa Fluor 488 conjugate (Molecular Probes Inc., Eugene, Oregon, USA). The slides were analyzed on a MultiProbe 2010 laser scanning confocal microscope from Molecular Dynamics (Sunnyvale, California, USA).

\section{Results}

DQ 65-79 does not inhibit expression or phosphorylation of the $I L-2 R$. We previously demonstrated that the DQ 65-79 peptide inhibited IL-2-mediated cell proliferation (15). The high-affinity IL-2R is composed of the $\alpha, \beta$, and $\gamma$ chains, which are upregulated to various degrees upon stimulation (21). Treatment of activated peripheral blood leukocytes with the peptide or rapamycin had no effect on the expression of any of these chains, as determined by flow cytometry, when compared with the DMSO media control or DQ 65-79(72D) control peptide (not shown). In addition, tyrosine phosphorylation $(22,23)$ of the $\beta$ and $\gamma$ chains was not affected by DQ 65-79, DQ 65-79(72D), or rapamycin (data not shown). Thus, the anti-proliferative effect of the peptide occurs downstream of IL-2R phosphorylation.

DQ 65-79 has no effect on general tyrosine phosphorylation or on nonreceptor tyrosine kinase activity. Although no effect of DQ 65-79 on IL-2R phosphorylation was detected, it was possible that other proximal events were affected by the peptide. Tyrosine phosphorylation of proteins is one of the earliest events to occur after activation (24). Phosphotyrosine proteins were immunoprecipitated from IL-2-stimulated T cells, and probed with anti-phosphotyrosine $\mathrm{Ab}$. No change in the pattern of phosphotyrosine proteins was observed with DQ 65-79 on IL-2-stimulated cells compared with controls (Figure 2a).

The Jak kinases, Jak 1 and Jak3, are key mediators of IL-2R signal transduction. In particular, Jak3, which is restricted in expression primarily to activated leukocytes, is critical for signaling, as demonstrated by the Jak $3^{-1-}$ mouse $(25,26)$. Jak 1 associates with the IL-2R $\beta$ chain, and Jak3 associates with the IL-2R $\gamma$ chain; both become tyrosine phosphorylated after activation of the receptor (27). No change in the phosphorylation of Jak1 or the association of Jak 3 with the IL-2R $\gamma$ chain were observed in cells treated with peptide (data not shown).

The Src kinase family members Lck and Fyn associate with the IL-2R $\beta$ chain $(28,29)$, and become phosphorylated upon activation of the receptor (30). Phosphorylation of these kinases can lead to the recruitment of other proteins involved in signaling, including PI 3-kinase $(31,32)$. Peptide treatment did not alter the amount of tyrosine-phosphorylated Fyn or Lck immunoprecipitated from activated cells (Figure $2 \mathrm{~b}$ ), or their kinase activity (Figure 2c). Therefore, treatment with DQ 65-79 does not inhibit phosphotyrosine activity in general, nor does it affect the activity of several proximal tyrosine kinases.

DQ 65-79 blocks p70 S6 kinase activity. We demonstrated that DQ 65-79 blocks events that are critical for cellcycle progression, with the most proximal event identified being p27 downregulation (15). Rapamycin also inhibits degradation of p27 (14) and other distal signaling molecules, including p70 S6 kinase $(33,34)$. This kinase is important, but not essential, for the induction of the proliferative response; cells lacking the p70 S6 kinase gene still proliferate, but at a slower rate (35). This kinase phosphorylates the ribosomal protein S6, leading to an increase in translation of ribosomal and elongation factor mRNAs (36). Rapamycin inhibits p70 S6 kinase activity indirectly by blocking the function of upstream mTOR, which directly phosphorylates p70 S6 kinase (37). Cells treated with DQ 65-79 or rapamycin exhibit impaired p70 S6 kinase activity (Figure 3). Peptide or rapamycin added directly to the

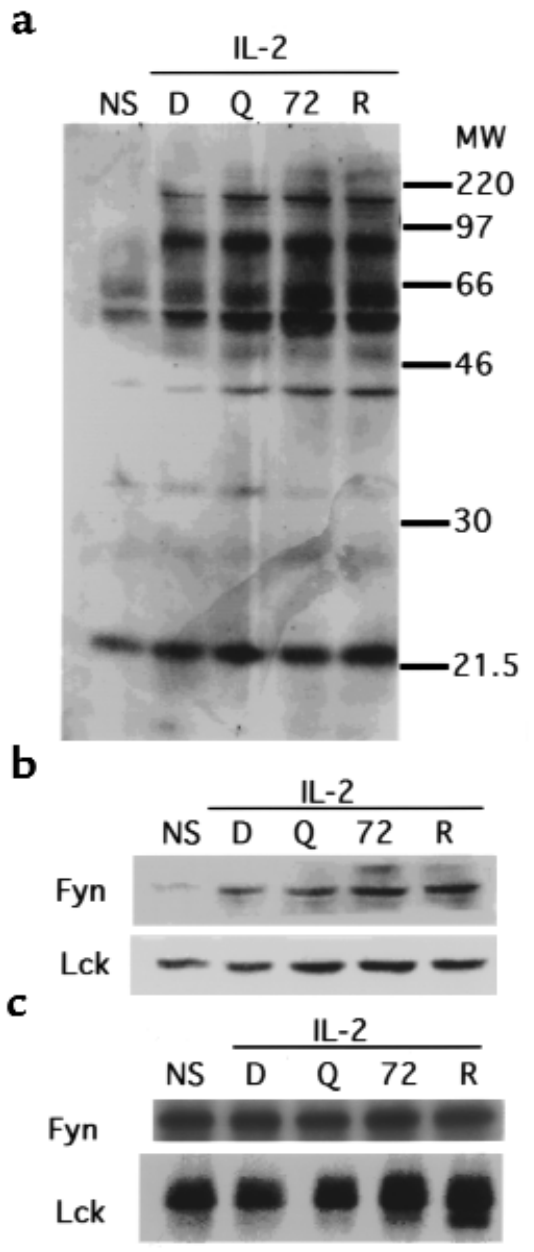

Figure 2

DQ 65-79 does not inhibit phosphotyrosine activity upon IL-2 stimulation. (a) Western blot using anti-phosphotyrosine mAb. (b) Western blot using anti-Fyn or anti-Lck. (c) Kinase activity of immunoprecipitated Fyn and Lck. NS, no stimulation; D, DMSO; Q, DQ 65-79; 72, DQ 65-79(72D); R, rapamycin. 


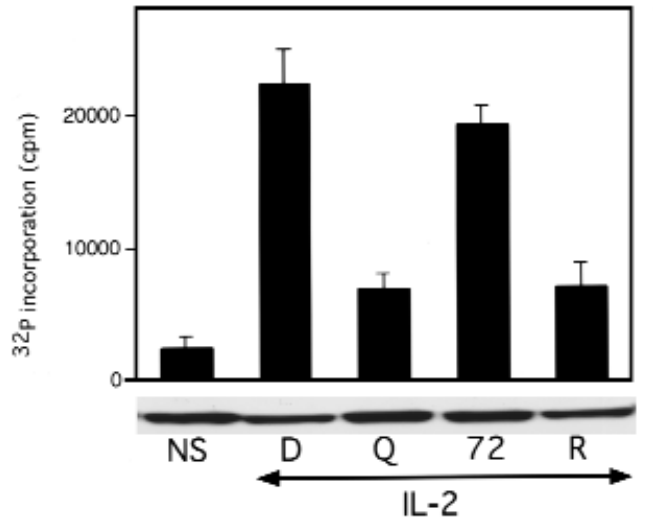

Figure 3

DQ 65-79 blocks p70 S6 kinase activity. Cells were stimulated with IL-2 in the presence of the indicated agents for 15 minutes, and p70 S6 kinase activity was determined. Western blot of p70 S6 kinase is shown below the kinase assay.

kinase assay had no effect (data not shown). Thus, another distal event in IL-2 receptor signaling is blocked by both DQ 65-79 and rapamycin.

Unlike rapamycin, DQ 65-79 inhibits Akt kinase activity. Akt, also known as protein kinase $B$, is a serine/threonine kinase in the IL-2R pathway that is not inhibited by rapamycin. Akt is involved in proliferation (38, $39)$, prevention of apoptosis $(39,40)$, and insulin signal transduction (41-43). The fungal metabolite wortmannin acts upstream of Akt, covalently binding to the p110 catalytic subunit of PI 3-kinase, blocking its kinase activity (44). Akt kinase activity was inhibited by treatment of cells with either DQ 65-79 or wortmannin, but not by rapamycin (Figure 4). This effect was indirect - peptide added to the kinase assay had no effect (data not shown). Thus, DQ 65-79 differs from rapamycin by blocking IL-2R signaling proximal to Akt.

$D Q$ 65-79 blocks PI 3-kinase activity in vitro. Intriguingly, residues 70-79 of DQ 65-79 are highly homologous to a region of the catalytic subunit of PI 3-kinase (Table 1), prompting us to examine the effect of DQ 65-79 on PI 3-kinase activity. PI 3-kinase is composed of a regulatory and a catalytic subunit, and there are several isoforms of each subunit (45). The regulatory subunit

Table 1

DQ 65-79 is homologous to the $\mathrm{p} 110$ subunit of PI-3 kinase

Sequence alignment

Identity Homology

(\%)

(\%)

DQ 65-79

PI-3K p 110- $\alpha$

$\mathrm{PI}-3 \mathrm{~K}$ p $110-\beta$

$\mathrm{PI}-3 \mathrm{~K} p 110-\gamma$

$\mathrm{PI}-3 \mathrm{~K} \mathrm{p} 110-\delta$

PI-4K 110

mTOR

K H N L N I V I K

$\underline{R} \quad H \quad N \quad S \quad N \quad I \quad \underline{M} \underline{V} K$

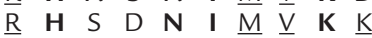

R H N D N I $\bar{M}$ I $T$

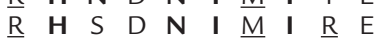

$R \quad H \quad N G N$ I $\bar{M} L \bar{D} K$

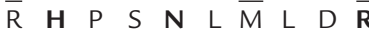

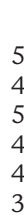

50
40
50
40
40
30

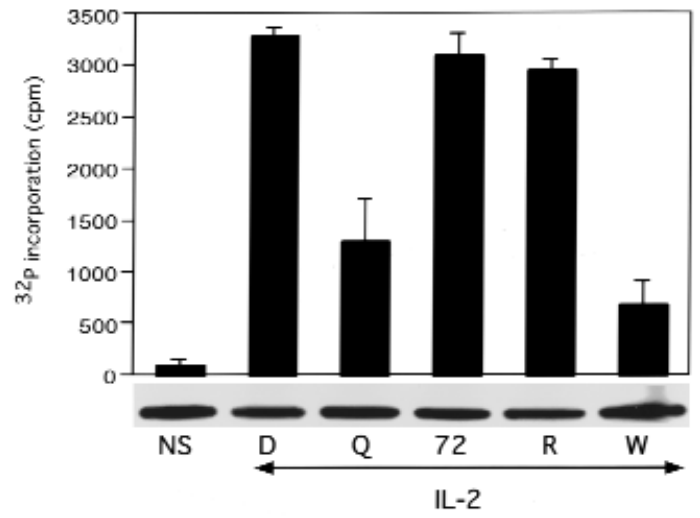

Figure 4

DQ 65-79 inhibits Akt kinase activity. Cells were stimulated with IL2 in the presence of the indicated agents for 15 minutes, and Akt kinase activity was determined. Western blot of Akt is shown below the kinase assay. W, wortmannin.

(p85) associates with the IL-2R upon stimulation, either directly or indirectly, by binding to phosphotyrosine residues (46). PI 3-kinase has been shown to be directly associated with the IL-2R $\beta$ chain (46), Fyn (31), Lck (32), IRS (47), and Grb2 (48). This association brings the catalytic (p110) subunit proximal to its lipid substrates (49). DQ 65-79 did not affect either subunit association or the interaction of the regulatory subunit with phosphotyrosine proteins (Figure 5).

Because DQ 65-79 did not alter the physical interactions of PI 3-kinase, we next tested the effects of the peptide on PI 3-kinase activity. A lipid kinase assay was performed with PI 3-kinase immunoprecipitated from peptide-treated cells. Whereas wortmannin blocked PI 3-kinase activity, no effect was detected with DQ 65-79 (Figure 6a). However, the effect of the peptide might not be apparent if the peptide did not bind directly to the kinase. The region of homology with the p110 subunit is in the catalytic part of the molecule rather than the region of subunit association (50), and the peptide has no effect on the association of the subunits. PI 3kinase activity was blocked when DQ 65-79 was added directly to the kinase assay (Figure 6b). Therefore, DQ 65-79 blocks the catalytic activity of PI 3-kinase in vitro in a manner distinct from wortmannin.

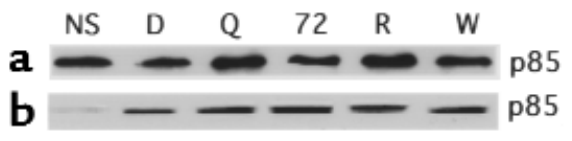

Figure 5

DQ 65-79 does not affect the association of the p85 and p100 subunits of PI 3-kinase or the association of $\mathrm{p} 85$ with tyrosine phosphorylated proteins. (a) The $\mathrm{p} 110-\beta$ subunit of PI 3 -kinase was immunoprecipitated from peptide-treated cells stimulated with IL-2, and the blot was probed with an mAb specific for p85. (b) Anti-phosphotyrosine $m A b$ was used to immunoprecipitate peptide-treated cells stimulated with IL-2. The blot was probed with anti-p85 mAb. 


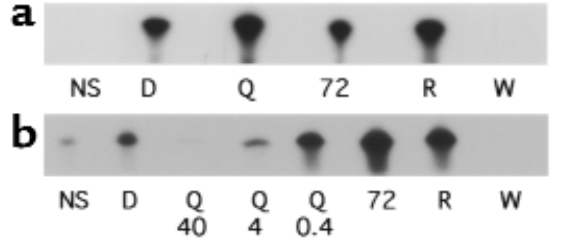

\section{Figure 6}

DQ 65-79 inhibits PI 3-kinase activity only when added directly to the in vitro lipid kinase assay. (a) Cells were stimulated with IL-2 in the presence of the indicated agents for 15 minutes, and a PI 3-kinase lipid kinase assay was performed. (b) PI 3-kinase was immunoprecipitated from IL-2-activated T cells, and DQ 65-79 (40 $\mu \mathrm{M}, 4 \mu \mathrm{M}$, and $0.4 \mu \mathrm{M})$, DQ 65-79(72D) $(40 \mu \mathrm{M})$, rapamycin (100 $\mathrm{nM})$, or wortmannin $(100 \mathrm{nM})$ was added directly to the lipid kinase assay.

DQ 65-79 crosses the cell membrane. These findings suggested that DQ 65-79 did not exert its effects by signaling through a membrane-bound receptor, but rather by directly inhibiting PI 3-kinase. We therefore asked whether DQ 65-79 enters cells to interact with PI 3-kinase. An analogue of DQ 65-79 was synthesized with an RYIRS tag on its carboxyl terminus (DQ 6579-IRS). This compound was indistinguishable from the untagged peptide in T-cell proliferation assays (data not shown). A cytolytic T-lymphocyte cell line was treated with DQ 65-79-IRS, permeabilized, and stained with both anti-IRS $\mathrm{mAb}\left(\operatorname{IgG}_{1}\right)$ and anti-HLA class I $\mathrm{mAb}\left(\operatorname{IgG}_{2 \mathrm{a}}\right)$. Isotype-specific secondary and tertiary antibodies conjugated with different fluorescent compounds were used to view localization of the peptide and HLA class I molecules by confocal microscopy (Figure 7). The anti-HLA class I staining was largely restricted to the cell membrane, appearing as a ring around the cells. In contrast, DQ 65-79-IRS had a very distinct staining pattern, and in many cells appeared to be present throughout the interior of the cell. Thus, DQ 65-79 enters the cell where it may associate with PI 3-kinase and inhibit its activity.

\section{Discussion}

A synthetic peptide corresponding to residues $65-79$ of the HLA class II allele $D Q A * 03011$ inhibits IL-2-mediated proliferation in a manner similar to that of rapamycin. DQ 65-79 does not affect the expression of the IL-2R or inhibit phosphorylation of the IL-2R $\beta$ chain. Although both DQ 65-79 and rapamycin inhibit p70 S6 kinase activity, only DQ 65-79 also blocks Akt kinase activity. In addition, there is striking homology between residues 70-79 of DQ 65-79 and the p110 subunit of PI 3-kinase. Whereas DQ 65-79 has no effect on the association of the two subunits of PI 3-kinase, it directly inhibits PI 3-kinase activity in vitro. Furthermore, confocal microscopy of cells treated with a tagged form of DQ 65-79 shows that it rapidly appears in the cytosol, where it can associate with PI 3-kinase. Thus, DQ 65-79 blocks the IL-2R signal transduction pathway by inhibiting PI 3-kinase activity.
Our initial hypothesis was that DQ 65-79 physically interfered with the interaction between $T$ cells and antigen-presenting cells by binding to the T-cell receptor. However, our findings do not support this. DQ 65-79 inhibits not only allorecognition but also proliferation of purified T cells in response to anti-CD3 or IL-2, indicating that antigen-presenting cells are not required for inhibition. Interestingly, the peptide does not inhibit PMA- and ionomycin-mediated proliferation (15), implying a membrane-associated effect. Additionally, DQ 65-79 inhibits intracellular signaling events in a manner similar to but distinct from the immunosuppressive drugs rapamycin and wortmannin, suggesting that DQ 65-79 acts within the cell. The observation that DQ 65-79 is rapidly internalized in cells (Drouvalakis and Krensky, manuscript in preparation) supports an intracellular locus for peptide action. Thus, the functional activities and physical location of DQ 65-79 are consistent with inhibition of PI 3-kinase activity.

Although the mechanism by which DQ 65-79 crosses the membrane has not been established, other peptides also translocate into cells. For example, conjugation of proteins to short cationic peptides enhances transport of proteins into cells $(51,52)$. Zhang and coworkers prepared cell-permeable synthetic peptides by incorporation of a variety of hydrophobic sequences (53). DQ 65-79 is mainly composed of hydrophobic and positively charged residues. Substitution analysis showed that replacement with serine at all but two of these residues resulted in a reduction of inhibitory activity (15). Thus, it is possible that the ability of DQ 65-79 to translocate into the cytosol is related to its overall hydrophobicity and positively charged amino acids.

Murphy and coworkers recently described a peptide designated HLA-DQA1 that inhibits allorecognition by inducing apoptosis (54). This peptide is derived from residues $62-77$ of the $\alpha$ chain of $\mathrm{DQA}^{*} 0101$, but differs

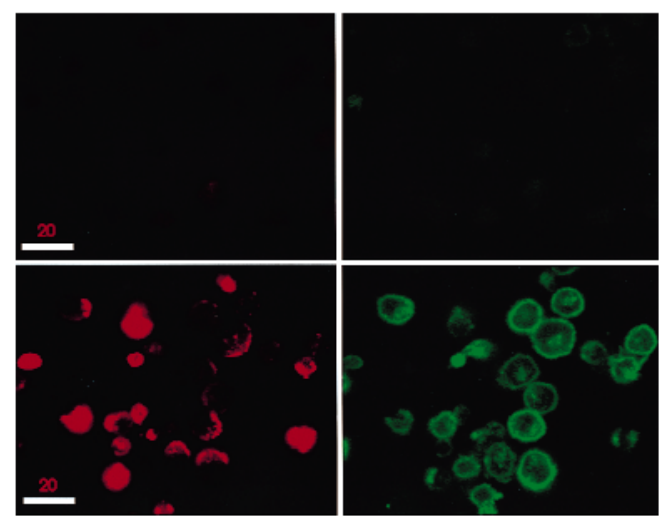

Figure 7

The DQ 65-79 peptide localizes both to the membrane and within the cell. Confocal microscopy of a cytotoxic T-cell line treated with DQ 6579-IRS and either class I MHC mAb (lower panels) or medium (upper panels) and processed as described in Methods. DQ 65-79 staining is red; class I staining is green. 
from DQ 65-79 in several ways: (a) HLA-DQA1 is three residues longer than DQ 65-79 at the amino terminus; (b) HLA-DQA1 is two residues shorter than DQ 65-79 at the carboxyl terminus; and (c) the core region of the two peptides differs at residues 66,69 , and 76 . It will be interesting to determine whether these two related peptides have similar mechanisms of action.

We have not yet determined exactly how DQ 65-79 interferes with PI 3-kinase activity. The region of homology between the peptide and p110 is within the catalytic region of the subunit that contains several functional subdomains (50). The DQ 65-79 homology region does not correspond to the ATP-binding region (amino acids 802-825) (50), to the covalent attachment site of wortmannin (amino acid 802) (55), or to the putative head binding region (amino acids 937-952) (56); rather, it corresponds to part of the kinase domain (amino acids 916-924) (50). Interestingly, a change at position 916 of $\mathrm{p} 110-\beta$ from arginine to lysine causes a complete loss of kinase function (57). The corresponding residue in DQ 65-79 is lysine instead of arginine, which may account for the inhibitory effects of the peptide. However, we are unable to demonstrate direct interaction between DQ 65-79 and PI 3-kinase, ATP, or the lipid substrate (Boytim and Clayberger, unpublished observations). The interaction of the peptide with the components of the in vitro kinase assay may be too weak to be detected by conventional methods. Alternatively (though it is not likely), the peptide may be acting through an associated protein that is coimmunoprecipitated with the kinase.

Due to the ubiquitous expression of PI 3-kinase and its role in many different signaling pathways, one might expect that DQ 65-79 would inhibit multiple cell types and multiple effector functions of those cells. We have observed a range in sensitivity to the antiproliferative effects of the peptide with different cell types, with lymphocytes being more sensitive than other types of cells. This may be attributed to the levels of different isoforms of PI 3-kinase, although it is not yet clear what role the different isoforms play in signaling specificity. It is intriguing that the $\mathrm{p} 110-\delta$ isoform is primarily leukocyte specific (58), which may provide an explanation for the more potent effects of DQ 65-79 on lymphocytes vs. other cell types.

DQ 65-79 may also inhibit the activity of other PI kinase family members. Wortmannin has been shown to block the kinase activity of PI 4-kinase (59), mTOR (60), and Vps34p (61). The PI kinase family member mTOR also has homology to DQ 65-79 in its kinase domain. Although no lipid kinase activity has been demonstrated for mTOR (56), the peptide may affect other activities of mTOR. Inhibition of multiple members of the IL-2R signaling pathway may explain the strong inhibitory effect of the peptide on proliferation, but the effect of the peptide on MTOR and other PI kinase family members remains to be investigated. It is also possible that the peptide may interact with other proteins within the cell. Therefore, although we have demonstrated the inhibition of PI 3-kinase as a primary action of DQ 65-79, other effects may also be involved in the inhibition of proliferation.

\section{Acknowledgments}

We thank Richard Roth for critical reading of this manuscript. This work was supported by National Institutes of Health grants AI-35125, DK-35008, AI-41520, and HD-34214. Alan M. Krensky is the Shelagh Galligan Professor of Pediatrics. Michelle L. Boytim was a Howard Hughes Medical Institute Predoctoral Fellow.

1. Germain, R.N. 1994. MHC-dependent antigen processing and peptide presentation: providing ligands for $\mathrm{T}$ lymphocyte activation. Cell. 76:287-299.

2. Lanier, L.L. 1997. Natural killer cell receptors and MHC class I interactions. Curr. Opin. Immunol. 9:126-131.

3. Lobo, P.I., and Patel, H.C. 1994. A novel role for MHC class II antigens: evidence implicating a protective effect on tumour cells against cytotoxicity by NK and LAK cells. Immunology. 83:240-244.

4. Foster, S., Wood, K.J., and Morris, P.J. 1989. Comparison of the effect of protein micelles containing purified class I MHC antigen and a cytosolic preparation containing water soluble class I molecules on rat renal allograft survival. Transplant. Proc. 21:375-376.

5. Pouteil, N.C., Wood, K.J., and Morris, P.J. 1993. The effect of purified class II major histocompatibility complex antigen on the survival of vascularized organ allografts in the rat. Transplantation. 55:656-658.

6. Nisco, S., et al. 1994. Induction of allograft tolerance in rats by an HLA class-I-derived peptide and cyclosporine A. J. Immunol. 152:3786-3792.

7. Buelow, R., Veyron, P., Clayberger, C., Pouletty, P., and Touraine, J.L. 1995. Prolongation of skin allograft survival in mice following administration of ALLOTRAP. Transplantation. 59:455-460.

8. Woo, J., Gao, L., Cornejo, M.C., and Buelow, R. 1995. A synthetic dimeric HLA class I peptide inhibits T cell activity in vitro and prolongs allogeneic heart graft survival in a mouse model. Transplantation. 60:1156-1163.

9. Cuturi, M.C., et al. 1995. Prolongation of allogeneic heart graft survival in rats by administration of a peptide (a.a. 75-84) from the alpha 1 helix of the first domain of HLA-B7 01. Transplantation. 59:661-669.

10. Sayegh, M.H., Khoury, S.J., Hancock, W.W., Weiner, H.L., and Carpenter, C.B. 1993. Induction of immunity and oral tolerance to alloantigen by polymorphic class II major histocompatibility complex allopeptides in the rat. Transplant. Proc. 25:357-358.

11. Sayegh, M.H., et al. 1993. Thymic recognition of class II major histocompatibility complex allopeptides induces donor-specific unresponsiveness to renal allografts. Transplantation. 56:461-465.

12. Murphy, B., Kim, K.S., Buelow, R., Sayegh, M.H., and Hancock, W.W. 1997. Synthetic MHC class I peptide prolongs cardiac survival and attenuates transplant arteriosclerosis in the Lewis->Fischer 344 model of chronic allograft rejection. Transplantation. 64:14-19.

13. Wiederrecht, G., Lam, E., Hung, S., Martin, M., and Sigal, N. 1993. The mechanism of action of FK-506 and cyclosporin A. Ann. NY Acad. Sci. 696:9-19.

14. Dumont, F.J., and Su, Q. 1996. Mechanism of action of the immunosuppressant rapamycin. Life Sci. 58:373-395.

15. Boytim, M.L., Lyu, S.C., Jung, R., Krensky, A.M., and Clayberger, C. 1998. Inhibition of cell cycle progression by a synthetic peptide corresponding to residues 65-79 of an HLA class II sequence: functional similarities but mechanistic differences with the immunosuppressive drug rapamycin. J. Immunol. 160:2215-2222.

16. Nourse, J., et al. 1994. Interleukin-2-mediated elimination of the p27Kip1 cyclin-dependent kinase inhibitor prevented by rapamycin. Nature. 372:570-573

17. Brennan, P., et al. 1997. Phosphatidylinositol 3-kinase couples the interleukin-2 receptor to the cell cycle regulator E2F. Immunity. 7:679-689.

18. Marshall, J.D., Wen, Y., Abrams, J.S., and Umetsu, D.T. 1993. In vitro synthesis of IL-4 by human CD4+ T cells requires repeated antigenic stimulation. Cell. Immunol. 152:18-34.

19. Elliott, L.H., and Levay, A.K. 1997. Costimulation with dexamethasone and prostaglandin E2: a novel paradigm for the induction of T-cell anergy. Cell. Immunol. 180:124-131.

20. Chin, J.E., Dickens, M., Tavare, J.M., and Roth, R.A. 1993. Overexpression of protein kinase $\mathrm{C}$ isoenzymes alpha, beta I, gamma, and epsilon in cells overexpressing the insulin receptor. Effects on receptor phosphorylation and signaling. J. Biol. Chem. 268:6338-6347.

21. Nakarai, T., et al. 1994. Interleukin 2 receptor gamma chain expression on resting and activated lymphoid cells. J. Exp. Med. 180:241-251. 
22. Asao, H., Kumaki, S., Takeshita, T., Nakamura, M., and Sugamura, K. 1992. IL-2-dependent in vivo and in vitro tyrosine phosphorylation of IL-2 receptor gamma chain. FEBS Lett. 304:141-145.

23. Mills, G.B., et al. 1990. Interleukin 2 -induced tyrosine phosphorylation. Interleukin 2 receptor beta is tyrosine phosphorylated. J. Biol. Chem. 265:3561-3567.

24. Farrar, W.L., and Ferris, D.K. 1989. Two-dimensional analysis of interleukin 2-regulated tyrosine kinase activation mediated by the p70-75 beta subunit of the interleukin 2 receptor. J. Biol. Chem. 264:12562-12567.

25. Thomis, D.C., Gurniak, C.B., Tivol, E., Sharpe, A.H., and Berg, L.J. 1995. Defects in B lymphocyte maturation and T lymphocyte activation in mice lacking Jak3. Science. 270:794-797.

26. Thomis, D.C., and Berg, L.J. 1997. Peripheral expression of Jak3 is required to maintain T lymphocyte function. J. Exp. Med. 185:197-206.

27. Miyazaki, T., et al. 1994. Functional activation of Jak1 and Jak3 by selective association with IL-2 receptor subunits. Science. 266:1045-1047.

28. Hatakeyama, M., et al. 1991. Interaction of the IL-2 receptor with the srcfamily kinase p56lck: identification of novel intermolecular association. Science. 252:1523-1528.

29. Kobayashi, N., et al. 1993. Functional coupling of the src-family protein tyrosine kinases p59fyn and p53/56lyn with the interleukin 2 receptor: implications for redundancy and pleiotropism in cytokine signal transduction. Proc. Natl. Acad. Sci. USA. 90:4201-4205.

30. Horak, I.D., et al. 1991. T-lymphocyte interleukin 2-dependent tyrosine protein kinase signal transduction involves the activation of p56lck. Proc. Natl. Acad. Sci. USA. 88:1996-2000.

31. Karnitz, L.M., Sutor, S.L., and Abraham, R.T. 1994. The Src-family kinase, Fyn, regulates the activation of phosphatidylinositol 3-kinase in an interleukin 2-responsive T cell line. J. Exp. Med. 179:1799-1808.

32. Taichman, R., Merida, I., Torigoe, T., Gaulton, G.N., and Reed, J.C. 1993. Evidence that protein tyrosine kinase p56-Lck regulates the activity of phosphatidylinositol-3'-kinase in interleukin-2-dependent T-cells. J. Biol. Chem. 268:20031-20036.

33. Dumont, F.J., Staruch, M.J., Koprak, S.L., Melino, M.R., and Sigal, N.H. 1990. Distinct mechanisms of suppression of murine T cell activation by the related macrolides FK-506 and rapamycin. J. Immunol. 144:251-258.

34. Kuo, C.J., et al. 1992. Rapamycin selectively inhibits interleukin-2 activation of p70 S6 kinase. Nature. 358:70-73.

35. Kawasome, H., et al. 1998. Targeted disruption of p70(s6k) defines its role in protein synthesis and rapamycin sensitivity. Proc. Natl. Acad. Sci. USA. 95:5033-5038.

36. Terada, N., et al. 1994. Rapamycin selectively inhibits translation of mRNAs encoding elongation factors and ribosomal proteins. Proc. Natl. Acad. Sci. USA. 91:11477-11481.

37. Burnett, P.E., Barrow, R.K., Cohen, N.A., Snyder, S.H., and Sabatini, D.M 1998. RAFT1 phosphorylation of the translational regulators p70 S6 kinase and 4E-BP1. Proc. Natl. Acad. Sci. USA. 95:1432-1437.

38. Reif, K., Burgering, B.M., and Cantrell, D.A. 1997. Phosphatidylinositol 3-kinase links the interleukin-2 receptor to protein kinase B and p70 S6 kinase. J. Biol. Chem. 272:14426-14433.

39. Ahmed, N.N., Grimes, H.L., Bellacosa, A., Chan, T.O., and Tsichlis, P.N. 1997. Transduction of interleukin-2 antiapoptotic and proliferative signals via Akt protein kinase. Proc. Natl. Acad. Sci. USA. 94:3627-3632.

40. Zhou, H., Summers, S.A., Birnbaum, M.J., and Pittman, R.N. 1998. Inhibition of Akt kinase by cell-permeable ceramide and its implications for ceramide-induced apoptosis. J. Biol. Chem. 273:16568-16575.

41. Kitamura, T., et al. 1998. Requirement for activation of the serine-threonine kinase Akt (protein kinase B) in insulin stimulation of protein synthesis but not of glucose transport. Mol. Cell. Biol. 18:3708-3717.

42. Scott, P.H., Brunn, G.J., Kohn, A.D., Roth, R.A., and Lawrence, J., Jr. 1998.
Evidence of insulin-stimulated phosphorylation and activation of the mammalian target of rapamycin mediated by a protein kinase B signaling pathway. Proc. Natl. Acad. Sci. USA. 95:7772-7777.

43. Kohn, A.D., Summers, S.A., Birnbaum, M.J., and Roth, R.A. 1996. Expression of a constitutively active Akt Ser/Thr kinase in 3T3-L1 adipocytes stimulates glucose uptake and glucose transporter 4 translocation. $J$. Biol. Chem. 271:31372-31378.

44. Thelen, M., Wymann, M.P., and Langen, H. 1994. Wortmannin binds specifically to 1-phosphatidylinositol 3-kinase while inhibiting guanine nucleotide-binding protein-coupled receptor signaling in neutrophil leukocytes. Proc. Natl. Acad. Sci. USA. 91:4960-4964.

45. Ho, L.K., Liu, D., Rozycka, M., Brown, R.A., and Fry, M.J. 1997. Identification of four novel human phosphoinositide 3-kinases defines a multiisoform subfamily. Biochem. Biophys. Res. Commun. 235:130-137.

46. Remillard, B., et al. 1991. Interleukin-2 receptor regulates activation of phosphatidylinositol 3-kinase. J. Biol. Chem. 266:14167-14170.

47. Myers, M., Jr., et al. 1994. Insulin receptor substrate-1 mediates phosphatidylinositol 3'-kinase and p70S6k signaling during insulin, insulinlike growth factor-1, and interleukin-4 stimulation. J. Biol. Chem. 269:28783-28789.

48. Wang, J., Auger, K.R., Jarvis, L., Shi, Y., and Roberts, T.M. 1995. Direct association of Grb2 with the p85 subunit of phosphatidylinositol 3kinase. J. Biol. Chem. 270:12774-12780.

49. Klippel, A., et al. 1996. Membrane localization of phosphatidylinositol 3-kinase is sufficient to activate multiple signal-transducing kinase pathways. Mol. Cell. Biol. 16:4117-4127.

50. Zvelebil, M.J., et al. 1996. Structural and functional diversity of phophoinositide 3-kinases. Philos. Trans. R. Soc. Lond. B. Biol. Sci. 351:217-233.

51. Ueda, Y., et al. 1995. Both CD28 ligands CD80 (B7-1) and CD86 (B7-2) activate phosphatidylinositol 3-kinase, and wortmannin reveals heterogeneity in the regulation of T cell IL-2 secretion. Int. Immunol. 7:957-966.

52. Bertrand, F., et al. 1998. A role for nuclear factor kappaB in the antiapoptotic function of insulin. J. Biol. Chem. 273:2931-2938.

53. Zhang, L., et al. 1998. Preparation of functionally active cell-permeable peptides by single-step ligation of two peptide modules. Proc. Natl. Acad. Sci. USA. 95:9184-9189.

54. Murphy, B., et al. 1999. Inhibition of allorecognition by a human class II MHC-derived peptide through the induction of apoptosis. J. Clin. Invest. 103:859-867.

55. Wymann, M.P., et al. 1996. Wortmannin inactivates phosphoinositide 3-kinase by covalent modification of Lys-802, a residue involved in the phosphate transfer reaction. Mol. Cell. Biol. 16:1722-1733.

56. Bondeva, T., et al. 1998. Bifurcation of lipid and protein kinase signals of PI3K gamma to the protein kinases PKB and MAPK. Science. 282:293-296.

57. Dhand, R., et al. 1994. PI 3-kinase is a dual specificity enzyme: autoregulation by an intrinsic protein-serine kinase activity. $E M B O \mathrm{~J}$. 13:522-533.

58. Chantry, D., et al. 1997. p110delta, a novel phosphatidylinositol 3-kinase catalytic subunit that associates with $\mathrm{p} 85$ and is expressed predominantly in leukocytes. J. Biol. Chem. 272:19236-19241.

59. Downing, G.J., Kim, S., Nakanishi, S., Catt, K.J., and Balla, T. 1996. Characterization of a soluble adrenal phosphatidylinositol 4-kinase reveals wortmannin sensitivity of type III phosphatidylinositol kinases. Biochemistry. 35:3587-3594.

60. Brunn, G.J., et al. 1996. Direct inhibition of the signaling functions of the mammalian target of rapamycin by the phosphoinositide 3-kinase inhibitors, wortmannin and LY294002. EMBO J. 15:5256-5267.

61. Volinia, S., et al. 1995. A human phosphatidylinositol 3-kinase complex related to the yeast $\mathrm{Vps} 34 \mathrm{p}-\mathrm{V} \mathrm{ps} 15 \mathrm{p}$ protein sorting system. EMBO J. 14:3339-3348. 